\title{
Reconstruir la identidad de aprendiz en la FP. El efecto de las experiencias escolares y las culturas de enseñanza en la construcción identitaria ${ }^{1}$
} Reconstructing Learner Identity in VET. The Effect of School Experiences
and Learning Cultures on Identity Construction

Aina Tarabini, Judith Jacovkis y Marta Curran²

\begin{abstract}
Resumen
La Formación Profesional en España sigue siendo una vía formativa escasamente desarrollada y prestigiada, a pesar de los importantes avances que se han registrado en los últimos años. El Grado Medio, en particular, presenta unos niveles de oferta y demanda muy inferiores a los de Bachillerato. Además, en términos globales, escolariza a un porcentaje mucho mayor de jóvenes de bajo estatus socioeconómico y cultural y con trayectorias de fracaso o desvinculación escolar durante la ESO. El objetivo de este artículo es analizar cómo construyen su identidad de aprendices los alumnos de FP en base a sus experiencias escolares y formativas -tanto anteriores como presentes- y a sus proyecciones de futuro. El análisis ha seguido una metodología cualitativa basada en 42 entrevistas en profundidad con estudiantes del primer curso de FP de Grado Medio en Barcelona. Los resultados muestran, por un lado, que la identidad de aprendices de los estudiantes de FP parte de una experiencia en la escolarización obligatoria mayormente negativa. Ésta, a su vez, se basa en una concepción pública de los mismos como malos estudiantes. Por otro lado, muestran la emergencia de procesos de resignificación de la identidad durante los estudios de FP basados en el reconocimiento y dignificación de los conocimientos prácticos que articulan las culturas de enseñanza y aprendizaje de esta vía formativa. En conjunto, el artículo contribuye a avanzar en el estudio de las identidades de los estudiantes como un elemento clave para comprender las desigualdades educativas.
\end{abstract}

\section{Palabras clave}

Identidad de aprendiz, experiencia escolar, culturas de enseñanza, formación profesional, desigualdad educativa.

\section{Abstract}

Upper Vocational Education in Spain remains as a poorly developed and prestigious training path, despite the significant progress made in recent years. Its levels of supply and demand are much lower than those of the Academic path -the Baccalaureate. In addition, in global terms, VET courses enrol a much higher percentage of working class' students as well as young people with trajectories of school failure. The objective of this article is to analyse how VET students construct their identity as learners based on their educational experiences - both past and present - and their projections for the future. The analysis develops a qualitative methodology based on 42 in-depth interviews with students in the first course of upper secondary VET in Barcelona. The results show, on the one hand, that the learner identity of VET students is constructed upon on a mostly negative experience of compulsory schooling. This, in turn, is grounded on a public conception of them as bad students. On the other hand, it proves the emergence of processes of identity ressignificance when they enrol in VET, based on the recognition and dignifying of the practical knowledge that articulates the teaching and learning cultures of this training path. In whole, the article contributes to advancing to the study of learner identities as a key element in understanding educational inequalities.

\section{Keywords}

Learner identity, school experience, learning cultures, vocational training, educational inequality.

\section{Cómo citar/Citation}

Tarabini, Aina; Jacovkis, Judith y Curran, Marta (2020). Reconstruir la identidad de aprendiz en la FP. El efecto de las experiencias escolares y las culturas de enseñanza en la construcción identitaria. Revista de Sociología de la Educación-RASE, 13 (4), 562-578. http://dx.doi.org/10.7203/ RASE.13.4.18047.

\footnotetext{
1 Este artículo se ha elaborado en el marco del proyecto I+D+I 'EDUPOST16. La construcción de las oportunidades educativas post-16. Un análisis de las transiciones a la educación secundaria postobligatoria en contextos urbanos (Ref. CSO2016-80004P. IP Aina Tarabini). Más información: http://edupost16.es.

2 Aina Tarabini, Universidat Autònoma de Barcelona, aina.tarabini@uab.cat; Judith Jacovkis, Universidat Autònoma de Barcelona, judith.jacovkis@uab.cat; Marta Curran, Universidat Autònoma de Barcelona, mcurran@ucm.es.
} 


\section{Introducción}

La teoría de las resistencias, emergente a finales de los años 70 del siglo pasado (Willis, 1997), fue la primera dentro de la tradición sociológica en incorporar el papel activo de los jóvenes para entender los procesos de reproducción y/o cambio social a través de la educación. Desde entonces, numerosas investigaciones sociológicas se han ocupado de estudiar las formas a través de las cuales la escuela produce identidades juveniles (Reay, 2010) y, a su vez, el papel crucial de dichas identidades en la comprensión de las desigualdades generadas en y a través de la educación.

Dichas investigaciones han producido información clave para entender cómo estas identidades están profundamente marcadas por la clase, el género y la etnicidad de los estudiantes (Archer y Yamashita, 2003; Hollingworth y Archer, 2010; Ingram, 2018; Reay et al., 2001). También han generado resultados fundamentales que explican que la propia construcción del alumno ideal no es neutra en términos de origen social. De hecho, las posibilidades de los jóvenes de concebirse como 'buenos estudiantes' no son independientes de los contextos sociales e institucionales en que se desarrollan. En este sentido, la literatura que se ha encargado de analizar de qué manera las prácticas pedagógicas y las concepciones docentes impactan sobre la construcción de la identidad de los jóvenes es diversa (Valenzuela, 1999). Tal como señalan Colley et al. (2003), las identidades de los jóvenes como estudiantes siempre se construyen en contextos institucionales específicos y en el marco de culturas de enseñanza y aprendizaje concretas (Brockmann, 2010; Ecclestone, 2007a; Hodkinson et al., 2007; Rees et al., 1997).

A pesar de ello todavía es escasa la literatura que analiza cómo la identidad de los estudiantes se construye progresivamente durante la trayectoria y la experiencia escolar; cómo ésta forma parte de un proceso de devenir (Colley, et al., 2003). Así mismo, tampoco abunda la investigación que analiza cómo esta identidad de aprendiz, en términos de Coll y Falsafi (2010), se configura en un elemento central para entender las trayectorias educativas de los jóvenes. De hecho, aunque numerosas investigaciones estudian cómo las identidades sociales de los jóvenes son proyectadas, reconocidas, omitidas y/o enaltecidas por la escuela, existen menos centradas en entender cómo contextos de aprendizaje específicos construyen una imagen de uno mismo en tanto que aprendiz (Coll y Falsafi, 2010). Y menos todavía las que estudian este tema en el contexto de la Formación Profesional (Brockmann, 2010).

En este contexto, el objetivo del artículo es analizar cómo construyen su identidad de aprendices los alumnos de Formación Profesional (FP) en base a sus experiencias educativas, pasadas y presentes, y a sus proyecciones de futuro. Partimos de la hipótesis de que esta identidad se construye en relación con experiencias educativas concretas que se generan en el marco de contextos instruccionales específicos. Asimismo, estas identidades son fundamentales para entender las trayectorias educativas en sentido amplio. Tal como afirman Harris y Rainey (2012), las trayectorias educativas de los estudiantes han sido mayormente explicadas a través de las oportunidades o constricciones generadas por diferentes arquitecturas institucionales. En este sentido, el papel de sus propias experiencias en la construcción de los itinerarios formativos ha tenido menos relevancia.

El análisis que planteamos no sólo entiende que la identidad se construye de forma secuencial y acumulativa y que, por tanto, está sujeta a cambios y contradicciones que es preciso identificar. La identidad es también relacional y se construye en base a atributos simbólicos que permiten definirse a uno mismo en relación con los otros. Es por ello, que para entender cómo se conforma la identidad de aprendiz de los estudiantes de FP hay que considerar los imaginarios y las representaciones públicas que se constru- 
yen alrededor de esta vía formativa. En este sentido, y adaptando la expresión de Hollingworth y Archer (2010) podemos afirmar que, en España, la FP sigue siendo un 'espacio educativo demonizado' que tiene menor prestigio y genera menos oportunidades que el Bachillerato (Tarabini y Jacovkis, 2019). En este sentido, entendemos que la naturaleza estratificadora del sistema educativo español configura las experiencias e identidades de los jóvenes.

El artículo, pues, representa una contribución a la comprensión de las desigualdades educativas y de las trayectorias educativas de los jóvenes a través del proceso de construcción de sus identidades de aprendices. Unas identidades que, como hemos señalado, se construyen en el marco de una experiencia de escolarización específica, en relación con universos simbólicos particulares y en el contexto de marcos institucionales específicos.

\section{Fundamentos teóricos}

La identidad es una categoría de carácter relacional basada en procesos de identificación y diferenciación de los otros. Hace referencia a quiénes somos en relación con el mundo, a nuestros umbrales simbólicos de adscripción y pertenencia (Valenzuela, 1997). Lejos de ser, por tanto, una esencia de los individuos es un proceso que se construye de forma progresiva, múltiple, y a menudo contradictoria, a través de relaciones sociales (Jenkins 1996). Las identidades siempre se producen en un marco de condiciones socio históricas particulares y, por ello, no se pueden entender al margen de las relaciones de poder y desigualdad (Archer y Francis, 2007). Como señalan Roseneil y Seymour (1999: 2, citados en Colley et al., 2003) 'no todas las identidades están igualmente disponibles para todos y no todas son igualmente valoradas'.

La escuela, en particular, tiene un rol central en la construcción de identidades. Como afirma Furlong (1991), se trata de una institución social que está fundamentalmente implicada en la producción de la juventud, en la construcción de su subjetividad y de su identidad pública. En este sentido, las normas, prácticas y expectativas escolares proporcionan materiales simbólicos clave a partir de los cuales los estudiantes dan sentido a sus experiencias y se definen a sí mismos (Perry, 2002, citado en Reay, 2010). De hecho, es numerosa la investigación sociológica que se ha encargado de señalar cómo la escuela, en tanto que institución, va transmitiendo la imagen del ‘buen alumno’ y los requisitos para el éxito escolar. Y en relación con esta imagen se va construyendo la propia identidad de aprendiz (Rees, et al., 1997; Hollingworth y Archer, 2010; Youdell, 2003). Es más, la imagen del alumno ideal se superpone con las características propias de determinados grupos sociales - chicas, blancas y de clase media profesional- y, precisamente por ello, es más fácil para unos que para otros construirse a sí mismos como 'buenos estudiantes'; porque las características de su posición social encajan mejor con la imagen dominante del 'alumno ideal' (Grant, 2006). Hay que tener en cuenta, además, que el concepto de identidad de aprendiz no puede entenderse sólo como un proceso cognitivo, sino como un proceso social, cultural y emocionalmente producido que está íntimamente relacionado con la autoestima (Hollingworth y Archer, 2010).

Las identidades de los estudiantes no se nutren sólo de sus propios rasgos y posiciones sociales sino que se desarrollan en contextos institucionales caracterizados por culturas de aprendizaje específicas. Como afirman Coll y Falsafi (2010) no sólo las identidades se construyen a través del aprendizaje, sino que también median la participación en el aprendizaje. En palabras de los autores 'el aprendizaje forma identidades y las identidades dan forma al aprendizaje' (Coll y Falsafi, 2010: 215). El concepto de cultura de aprendizaje (Hodkinson et al., 2007), de hecho, ha permitido desarrollar un conjunto muy significativo 
de investigaciones que entienden la construcción de las identidades en el marco de experiencias de aprendizaje particulares y de estructuras de oportunidades de aprendizaje concretas (Brockmann, 2010; Colley et al., 2003; Ecclestone, 2007a). Como señalan Rees et al. (1997: 490) 'la capacidad de un individuo para aprovechar las oportunidades de aprendizaje disponibles está limitada por su historia previa en este ámbito'. Asimismo, la estructura de oportunidades de aprendizaje a la que tienen acceso los individuos difiere enormemente en función de marcos históricos, territoriales e institucionales específicos. En este sentido, la investigación de Colley et al. (2003) demuestra que las culturas dominantes de enseñanza y aprendizaje en la FP contribuyen a definir un habitus profesional específico entre los estudiantes, orientado a cumplir los requisitos de una fuerza de trabajo futura obediente y disciplinada. De hecho, diversos estudios (Nylud y Rosvall, 2016; Wheelahan, 2007) muestran que las reformas recientes de FP en diferentes países han construido un currículum cada vez más técnico, enfocado a la adquisición de competencias concretas y fácilmente transferibles al mercado de trabajo. Y este currículum incorpora de forma implícita una definición de cómo se debe ser y cómo se debe aprender en este itinerario formativo.

En este mismo sentido, la investigación biográfica de Brockmann (2010) muestra que las experiencias escolares negativas de numerosos estudiantes les hacen desarrollar una identidad de aprendices basada en el rechazo de estilos particulares de enseñanza y aprendizaje. De hecho, en su estudio se pone de manifiesto que la elección de FP está, en numerosas ocasiones, vinculada con la oposición a formas académicas y teóricas de acceso al conocimiento. A pesar de ello, la autora afirma que este proceso no es el resultado de una actitud pasiva por parte de los estudiantes, sino que, al contrario, es a través de una disposición activa que construyen su identidad de aprendices ligada a aspectos manuales y prácticos. Según afirma la autora, estos jóvenes -lejos de los estereotipos acerca de los estudiantes de FP- no se conciben a sí mismos como estudiantes de segunda ni como fruto del fracaso escolar.

La construcción de la identidad de aprendiz está imbricada en las trayectorias educativas, laborales y vitales de los jóvenes. Investigaciones como las de Archer y Yamashita (2003), Ball et al. (2000), Ecclestone (2007b) o Reay et al. (2001) en Reino Unido muestran que las elecciones educativas de los jóvenes no se explican como resultado de cálculos racionales individuales ni tampoco por el efecto aislado de elementos estructurales y/o institucionales. Es en la conexión entre estructura y agencia y, por tanto, en la construcción de las identidades donde deben entenderse dichos procesos de elección. Por ello, la elección de itinerarios profesionales se vincula inexorablemente con la percepción y el sentido de uno mismo, con las experiencias escolares pasadas y con las proyecciones de futuro. En términos de Ecclestone (2007a), los estudiantes eligen caminos escolares que reflejan y refuerzan una imagen particular de sí mismos en tanto que tipos específicos de aprendices 'adecuados' para contextos educativos particulares.

\section{Contexto}

La educación secundaria en España se divide en dos etapas. La primera, de Educación Secundaria Obligatoria (ESO) tiene una duración de 4 cursos (de los 12 a los 16 años). Aunque formalmente es comprehensiva, en la práctica da cabida a distintas formas de adaptación curricular que van desde la agrupación por niveles dentro del centro hasta el uso de Unidades de Escolarización Compartida, situadas fuera del mismo y en las que determinado alumnado pasa un número variable de horas en los dos últimos cursos de la ESO. Una vez obtenido el Graduado en ESO (GESO), el alumnado puede acceder a uno de los dos itinerarios de la segunda etapa de educación secundaria: el académico (Bachillerato) o el profesional (Formación Profesional de Grado Medio, en adelante FP). Cada uno de los itinerarios tiene una duración 
de dos cursos. Desde Bachillerato se puede acceder tanto a la Universidad (mediante una prueba de acceso) como a la FP de Grado Superior. Desde la FP de Grado Medio, después de una prueba de acceso, se puede acceder al Grado Superior.

La FP ha experimentado cambios profundos en los últimos 20 años que han estado, en parte, orientados a mejorar su imagen en términos de prestigio social (García Jiménez y Lorente García, 2015). En la actualidad, sin embargo, este itinerario formativo sigue siendo representado como una vía de segunda en el imaginario social. Por un lado, la FP atrae a una proporción del alumnado menor que el Bachillerato (34,45\% en el curso 2018-19), y con una complejidad social mayor. Por el otro, tal y como señala Brockmann (2010) para el caso inglés, los conocimientos que se asocian a la vía académica (Tarabini y Jacovkis, 2020) siguen monopolizando prácticamente todas las materias que se imparten durante la ESO. Ello refuerza, desde la propia institución escolar, una jerarquía entre conocimientos en la que los saberes prácticos o aplicados se sitúan por debajo de los teóricos o abstractos, que siguen conformando lo que Raffe (1992) denomina como 'traditional academic mainstream'.

En cualquier caso, una vez acceden a la FP, los estudiantes encuentran un modelo de enseñanza-aprendizaje que difiere en varios aspectos del que se despliega mayoritariamente en la ESO y en el Bachillerato. En la vía profesional, el currículo se organiza en base a módulos formativos -y no a asignaturas- que responden a una definición de competencias profesionales que se realiza a nivel estatal. Esta organización evidencia la voluntad de conectar de forma directa la FP con el mundo laboral a través de los conocimientos y competencias que se trabajan en el aula y se exigen al alumnado. En sintonía, el tiempo se distribuye entre actividades de carácter teórico y otras de carácter práctico, pero todas orientadas al desarrollo de las competencias profesionales vinculadas a cada especialidad formativa así como de competencias transversales sobre conocimiento del mercado laboral. Finalmente, el alumnado realiza unas prácticas profesionales en el centro de trabajo ${ }^{3}$ con las que se busca profundizar sus competencias profesionales y dotarle de un conocimiento y de una experiencia sobre el funcionamiento real de la profesión en la que se está formando.

En conjunto, el modelo enseñanza-aprendizaje que se despliega en la FP está ligado a una experiencia profesional específica y conjuga conocimientos -teóricos y prácticos- ligados a ella y a los hábitos de trabajo que requerirá el alumnado una vez entre en el mercado laboral. Las dimensiones cognitiva y conductual que se desarrollan en esta modalidad formativa contribuyen a desarrollar en el alumnado una cultura profesional (Frykholm y Nitzler, 1993, citados en Colley et al., 2003) que lo vincula -también emocionalmente- con la profesión para la que se está formando.

\section{Metodología}

Estudiar las identidades de los jóvenes implica necesariamente un enfoque metodológico cualitativo capaz de adentrarse en las experiencias que éstos ofrecen de su proceso educativo y de captar las estructuras de sentido y significado que se esconden bajo estas explicaciones. De hecho, las identidades se forman en y a través del discurso. Los discursos, lejos de ser meramente descriptivos, tienen un fuerte componente productivo (Hollingworth y Archer, 2010). Es decir, permiten generar marcos de significado; de adscripción o exclusión. Y es por ello que identidades y discursos están profundamente imbricados y son

3 En la modalidad Dual se amplían las horas que el alumnado pasa en el centro de trabajo, pero el centro educativo sigue siendo central para el desarrollo de las competencias profesionales, aunque se espera que de forma más coordinada con el tejido empresarial. 
inseparables de las relaciones de poder. Como afirma Ball (1993), el discurso ejerce poder a través de la producción de conocimiento y 'verdades'. En este sentido, aproximarse a las identidades de aprendices de los jóvenes implica entender cómo se significan discursivamente en lugares y tiempos específicos; cómo se posicionan discursivamente en relación con los otros.

Para realizar este artículo, en concreto, se han realizado 42 entrevistas en profundidad a estudiantes del primer curso de FP de Grado Medio en 8 centros educativos de Barcelona ${ }^{5}$. La selección de la muestra responde a criterios de heterogeneidad, tanto en términos de género, como de origen social y de modalidad formativa. A pesar de ello, tal como refleja la Tabla I, la muestra reproduce la sobrerrepresentación de estudiantes de bajo estatus socioeconómico y cultural en esta modalidad formativa ${ }^{6}$.

Tabla I. Distribución de la muestra de estudiantes según familia profesional, género y clase social

\begin{tabular}{lcccc}
\hline & GÉNERO & & CLASE SOCIAL $^{7}$ \\
\hline FAMILIA PROFESIONAL & CHICAS & CHICOS & CT \\
\hline Administración y gestión & 2 & 2 & & 4 \\
\hline Actividades físicas y deportivas & 2 & 2 & & 4 \\
\hline Comercio y marketing & 2 & 2 & 2 & 6 \\
\hline Electricidad y electrónica & 2 & 4 & 4 & 2 \\
\hline Imagen personal & 2 & 2 & 4 \\
\hline Informática y comunicación & 2 & 2 & & 4 \\
\hline Sanidad & 4 & 4 & & 4 \\
\hline Servicios socioculturales y a la comunidad & 2 & 2 & 6 & 4 \\
\hline Transporte y mantenimiento de vehículos & 1 & 3 & & 36 \\
\hline Total & 19 & 23 & & 4 \\
\hline
\end{tabular}

Fuente: elaboración propia.

Las entrevistas han sido transcritas literalmente y codificadas con Atlas.Ti. Para este análisis, se han explotado las citas agrupadas en los códigos 'identidad', 'perfil social' y 'trayectoria educativa'. El primero comprende las referencias explícitas al 'yo', en tanto que reflejo de la 'identidad personal' y las menciones directas e indirectas al 'nosotros' y al 'ellos', en tanto que reflejo de la 'identidad o la contra-identidad colectiva'. Las referencias a la identidad colectiva se han explorado tanto en relación a aspectos sociales (principalmente, el género, la etnia, y la clase social) como educativos (fundamentalmente, la modalidad formativa). El 'perfil social' incluye la información sobre las características sociales de la familia -capital económico, cultural y social, y estructura familiar- y del barrio de residencia. Finalmente, la 'trayectoria educativa' incorpora la información sobre la trayectoria educativa desde primaria hasta la actualidad, tanto en términos descriptivos como respecto a la relación que tiene el alumno con las dimensiones cognitiva, conductual y emocional de la institución escolar.

Los discursos codificados se han analizado a la luz de las variables que conforman el modelo de análisis específico del artículo (Tabla II), y que sintetizan los elementos cruciales que apunta la literatura especializada sobre el proceso de construcción de identidades en general, y sobre las identidades de los

4 Se han seleccionado estudiantes de primer curso de FP con el propósito de explorar el proceso de transición entre la educación obligatoria y la postobligatoria, siendo éste uno de los principales objetivos del proyecto de investigación en el que se enmarca el artículo.

5 Todos los centros seleccionados se caracterizan por disponer de una oferta completa de educación secundaria (ESO, Bachillerato y FP), lo que los diferencia de la mayoría de centros de la ciudad. Además de esta especificidad, para garantizar la heterogeneidad de la muestra se han aplicado dos criterios complementarios: la composición social de los centros y la oferta de diferentes modalidades formativas tanto de Bachillerato como de FP.

6 La heterogeneidad en el perfil de los jóvenes de la muestra permite profundizar en la configuración de las identidades de aprendices los estudiantes de FP desde una perspectiva comparativa. Este artículo, sin embargo, por cuestiones de espacio y objetivo, no analiza la relación entre las diferentes variables de estudio (género, modalidad formativa o centro de escolarización) y la diversidad de formas a través de las cuales se despliega dicha identidad.

7 La variable de la clase social se ha construido de acuerdo con el nivel de estudios de los progenitores y sus respectivas ocupaciones. 
estudiantes en particular. Entendemos que su sistematización representa un valor añadido para avanzar en el desarrollo empírico de este objeto de estudio.

Tabla II. Modelo analítico para el estudio de la identidad

\begin{tabular}{|c|c|}
\hline \multirow{3}{*}{ SECUENCIAL } & Se construye en relación con el pasado. \\
\hline & Se reconstruye en el presente. \\
\hline & Se proyecta en el futuro. \\
\hline \multirow{2}{*}{ ACUMULATIVA } & Se nutre de experiencias y prácticas formativas pasadas (que pueden ser contradictorias) \\
\hline & Se reconstruye y expresa como una narración coherente (selección de experiencias, que puede variar en el tiempo) \\
\hline \multirow{2}{*}{ RELACIONAL } & Se construye en relación con los otros \\
\hline & Es construida por la visión de los otros \\
\hline \multirow{2}{*}{$\begin{array}{l}\text { SOCIAL E INSTITUCIONALMENTE CONSTRUIDA } \\
\text { (TAMBIÉN CULTURAL, HISTÓRICA, POLÍTICA) }\end{array}$} & Se imbrica con las características de la posición social en términos de clase social, género y etnicidad. \\
\hline & Se construye en relación con las características sociales, pedagógicas y curriculares de los centros educativos. \\
\hline \multirow{3}{*}{ MÚLTIPLE } & Elementos cognitivos. \\
\hline & Elementos conductuales. \\
\hline & Elementos emocionales. \\
\hline \multirow{2}{*}{ EXPRESADA/PRODUCIDA POR } & Discursos \\
\hline & Prácticas \\
\hline
\end{tabular}

Fuente: elaboración propia.

\section{Resultados}

\subsection{Identidades deterioradas: la imposibilidad de sentirse exitoso en la ESO}

Las identidades de aprendices del alumnado de FP se configuran a partir de una experiencia educativa en la ESO marcada, usualmente, por trayectorias de notas bajas, suspensos, repeticiones y, en la mayoría de ocasiones, por su asignación a grupos de refuerzo, a programas de adaptación curricular o a grupos de nivel bajo. De hecho, los jóvenes entrevistados afirman que su paso por dispositivos de adaptación curricular durante la ESO ha limitado sus oportunidades educativas futuras, tanto por lo que se refiere a la adquisición de conocimientos como a las posibilidades de acceso a la educación postobligatoria.

No tengo tan buen nivel de matemáticas como bubiera podido tener si no bubiera ido al Aula Abierta (grupo adaptado). (Pere, Auxiliar de Enfermería).

En el grupo $C$ todos íbamos a Ciclos, si querías hacer Bachillerato tenias que estar en el grupo $A$ o en el B pero en el $C$ no se puede hacer Bachillerato. (Omid, Peluquería).

Esta experiencia escolar genera una identidad de aprendiz frágil (Brockmann, 2010) que dificulta la concepción de uno mismo como 'buen estudiante' y lleva a procesos de interiorización del fracaso escolar. Tal como muestran investigaciones previas en este campo (Archer y Yassamita, 2003; Hollingworth y Archer, 2010; Valenzuela, 1999) aquellos que han vivido experiencias escolares negativas durante su trayectoria educativa tienden a culparse a sí mismos de su propio fracaso. Y consecuentemente se definen a sí mismos como menos listos y menos capaces que otros ${ }^{8}$. De hecho, los procesos crecientes de individualización dificultan enormemente la construcción de un relato sobre estas

8 En este punto vale la pena señalar que nuestros resultados difieren de los de Brockmann (2010). Como veremos en el próximo apartado, aunque algunos alumnos consiguen, a través de su experiencia en la FP, resinificar de forma activa su identidad como aprendices, su paso por la ESO y el fracaso acumulado que han experimentado durante esta etapa educativa sí que se interioriza como un fracaso propio. 
trayectorias que se aleje de la auto-culpabilización (Ball et al., 2000) y explican, tal como argumentan Biggart y Furlong (1996), por qué hoy en día es mucho más difícil encontrar formas de resistencia escolar cómo las que identificó Willis en sus estudios de los años 70.

Bueno, todos nos parecemos porqué somos los que siempre nos han dicho que no valemos y bueno muchos somos repetidores y eso. (Alex, Auxiliar de Enfermería).

Esta experiencia escolar negativa, además, se nutre en el presente de la participación en un itinerario formativo que, como hemos argumentado, todavía está fuertemente desprestigiado. Y esta construcción social de la FP como 'espacio educativo demonizado' (Hollingworth y Archer, 2010) tiene un claro impacto en la configuración de las identidades de aprendices de los estudiantes. De hecho, los jóvenes entrevistados son plenamente conscientes de que el itinerario en que están escolarizados se constituye en el imaginario colectivo como una vía de 'segunda' para aquellos que no están 'preparados' o 'capacitados' para hacer Bachillerato. Es más, de los relatos de los jóvenes se desprende el papel fundamental que juegan los docentes de la ESO en la construcción de esta imagen negativa de la FP. Ello contribuye a reforzar los resultados de las investigaciones previas que alertan sobre los sesgos de los procesos de orientación escolar y sobre la necesidad de abordarlos como una tecnología clave de poder y disciplinamiento social (Romito, 2017). Como vemos en las siguientes citas, los estudiantes relatan la frustración que les supone que les hayan orientado hacia una vía formativa que se plantea, tanto material como discursivamente, en términos negativos:

\section{Parece que los Grados Medios sean para los desterrados. Hay un tabú por asi decirlo sobre los Ciclos. [Los profesores de la ESO] hablaban mejor del Bachillerato y el Grado Medio era para la gente a la que le costaba estudiar. A mi no me preguntaron si quería hacer Bachillerato, a mi directamente me dijeron: Tú, a Grado Medio. (Lorena, Auxiliar de Enfermería).}

Este desprestigio no es sólo transmitido por parte de las autoridades pedagógicas -tutores y orientadores-, sino que también se ve reforzado por su grupo de iguales. Así, a estudiantes como Edgar, sus compañeros los conciben como 'malos estudiantes' por el hecho de haber sido orientados a FP.

Gran parte de mis compañeros iban a hacer Bachillerato. Cuando yo les decía que quería hacer un Ciclo, que era la única opción que me daban, ellos me decian 'qué mal tío, los Ciclos son para las personas que no estudian’. (Edgar, Instalaciones Eléctricas y Automáticas).

De hecho, la construcción de la identidad de aprendiz de los estudiantes de FP no puede entenderse al margen de la construcción de la identidad de los estudiantes de Bachillerato. Es más, una se construye y se produce en oposición a la otra. Los estudiantes de Bachillerato son considerados 'listos', 'esforzados', aquellos a quienes les gusta o no les cuesta estudiar. Y por contraste, los estudiantes de FP construyen un fuerte sentido de identidad como aprendices de segunda (Eccelstone, 2007a). En esta línea, el alumnado de FP no sólo se describe a partir de los atributos que percibe tener sino, y sobre todo, a partir de lo que lamenta carecer. Estos estudiantes no disponen de los atributos cogniti- 
vos o actitudinales que les hagan aptos para cursar la vía formativa que abre las puertas a la movilidad social. Y por ello desarrollan una identidad deteriorada (Hollingworth y Archer, 2010).

\section{P. ¿Dirías que hay unos estudios que son más adecuados para unos estudiantes que para otros? R. Sí. Por ejemplo, las personas de inteligencia superior que las demás, pueden hacer estudios más avanzados y pueden acceder a muchas clases de trabajo. P. Entonces es un tema que depende de la inteligencia. R. Sí. (Manel, Auxiliar de Enfermería).}

Bacbillerato es para los inteligentes. (Paula, Auxiliar de Enfermería).

Esta 'identidad deteriorada' se expresa a través de las múltiples dimensiones que configuran tanto la identidad como la vinculación escolar. A saber, la cognitiva, la conductual y la emocional. Desde un punto de vista cognitivo, el alumnado de FP muestra una falta de confianza en sus habilidades, siente que no sirve para estudiar, que no se le da bien, que le cuesta concentrarse y que saca malas notas. Así, en las descripciones que hacen de sí mismos como aprendices tienden a aparecer, a menudo de forma esencialista, atributos como 'lento', 'malo', 'normal', 'regular'. Además, estas descripciones los alejan de materias centrales en la ESO -y en Bachillerato- como son las lenguas o las matemáticas. Estas materias se asocian fuertemente con el conocimiento abstracto y se relacionan con un estilo particular de aprendizaje que se caracteriza por una enseñanza directiva y poco aplicada.

Yo tengo una forma de aprender diferente, algo más lento, más tranquilo, porque sino me agobio y no lo entiendo, y abi es cuando te bloqueas y no quieres entender nada. (Alexia, Electromecánica de Vehículos).

Me defino como un estudiante regular, soy normal, pasable. (Nala, Instalaciones Eléctricas y Automáticas).

No era buen estudiantes. Soy malo en lenguas. (Fernando, Sistemas Microinformáticos y Redes).

Los números y yo somos incompatibles. (Laura, Estética y Belleza).

Desde el punto de vista conductual, los relatos de los jóvenes entrevistados proyectan una imagen de sí mismos como no adaptados a la norma escolar o a la gramática escolar dominante en los centros de ESO. En este sentido, se autodefinen como vagos, con dificultades para estar muchas horas sentados y atender concentrados a la explicación de los docentes. Así, es imprescindible señalar que la definición de lo que es una buena conducta escolar, y la definición de lo que representan el mérito y el esfuerzo, no son elementos objetivos, naturales y/o vinculados de forma inherente a la acción de los individuos, sino que son construcciones sociales y culturales que, como tales, están profundamente atravesadas por los ejes de desigualdad social (Youdell, 2003). 


\section{P. ¿Cómo definirias al estudiante de FP? R. Seguramente como alguien a quien no le guste} mucho estar todo el rato con un libro o tomando apuntes, alguien que prefiera estar en contacto directo con el medio de trabajo. (Pere, Auxiliar de Enfermería).

Finalmente, y desde el punto de vista emocional, las entrevistas muestran que la experiencia de estos estudiantes durante la ESO se caracteriza por una sensación de no encajar y de no sentirse identificados con lo que se estaba enseñando. Como veremos en el próximo apartado, la vinculación emocional con los estudios que se están cursando es un elemento clave de construcción de la identidad de aprendiz. Esta vinculación está, a su vez, conectada con la valoración y el reconocimiento de otros acerca de las aptitudes y actitudes propias. Por ello, las dificultades para transitar con éxito por la ESO, y la consideración de que para otros este es el camino fácil, provocan a menudo un proceso de auto-exclusión que hace que la opción del Bachillerato ni siquiera se plantee como posible.

\section{P. ¿Por qué Ciclos y no Bachillerato? R. Porque me veía incapaz: (Vicent, Sistemas} Microinformáticos y Redes).

\subsection{Resignificación de la identidad: la FP como espacio de refugio y reconocimiento}

La identidad de aprendiz se nutre de experiencias educativas pasadas -y de su selección y reconstrucción a posteriori-, de experiencias educativas presentes, y de proyecciones de futuro que las dotan de sentido (Coll y Falsafi, 2010). Tal como hemos visto, la experiencia de los estudiantes de FP durante la ESO tiene claras connotaciones negativas -fracaso, imagen de mal estudiante, comparación con Bachillerato- que les alejan de una construcción mayoritaria de su identidad como ‘buenos alumnos'.

El acceso a la FP representa para muchos estudiantes una reconversión de su propia imagen como aprendices y de sus relaciones con la institución educativa ${ }^{9}$. En este sentido, merece la pena subrayar tres elementos que nos ayudan a entender cómo se modifica la propia imagen de aprendiz a través de un proceso de incorporación de un habitus profesional (Colley et al., 2003), que se vincula con las características diferenciales de la FP respecto de la ESO y con el tipo de relaciones que se establecen con los pares (Ecclestone, 2007a).

En primer lugar, el alumnado de FP se identifica con un conocimiento más práctico o aplicado que el que se prioriza durante la ESO. En sus discursos, ello sirve tanto para reflexionar sobre las causas de su desafección escolar en la etapa anterior como para explicar su mayor vinculación con los estudios profesionales. Por un lado, muchas de las entrevistas indican un reposicionamiento respecto a su imagen de 'mal estudiante' que se produce al ver reconocidas en la FP sus habilidades en 'lo práctico'. De algún modo, este reconocimiento permite a los entrevistados rehacer las nociones de habilidad, capacidad, inteligencia o maña de forma que sus propias experiencias escolares - previas y presentescontribuyen a mejorar la imagen que tienen de sí mismos y de la opción formativa que han elegido, y a modificar su identidad de aprendiz vinculándola con una imagen más positiva de sí mismos.

9 No tenemos espacio aquí para desarrollar las distintas formas de identificación que construye el alumnado con los estudios profesionales. Por ello, en este apartado nos centramos únicamente en los discursos de aquéllos que muestran una reconversión de la identidad de aprendiz vinculada con su acceso a la FP. 
En Bachillerato te hacen aprendértelo todo, y luego se te va a olvidar y no hacen nada de práctica. Yo creo que se aprende más haciendo práctica, que aprendiéndote un párrafo. (Blas, Electricidad).

Por otro lado, la orientación aplicada de la FP dota de un sentido más amplio al proceso educativo como preparación para el mercado de trabajo. Como hemos mostrado en el anterior apartado, durante la ESO y en la transición a la educación secundaria postobligatoria se tiende a asociar inteligencia con Bachillerato y falta de validez con FP. En cambio, la siguiente cita muestra que en el contexto de la FP se valora otro tipo de conocimiento que, en palabras de Ecclestone (2007a) se configura como una 'zona de confort' para los jóvenes. También puede entenderse como un 'acto de reconocimiento' en términos de Coll y Falsafi (2010). Esta zona de confort es la que permite concebir la maña y la destreza como conocimiento y, por tanto, atribuirle un valor simbólico a partir del cual los estudiantes pueden construir una nueva imagen de sí mismos como alumnos educables o exitosos (Hollingworth y Archer, 2010). A pesar de ello, no consigue desarticular la idea dominante de que diferentes estudiantes están 'dotados' de forma casi innata de diferentes tipos de conocimientos y que dichos conocimientos se asocian de forma mecánica con las diferentes vías formativas postobligatorias.

\section{P. ¿ंTú crees que son como robots en Bachillerato? R. Sí. Tú puedes ser tonto, tener un coeficiente intelectual bajo, pero si te tiras estudiando toda la tarde, al final te lo vas a sa- car. P. ¿En FP crees que no podría pasar? R. Aqui, en parte es por la parte práctica, tú puedes sacar dieces en los exámenes, pero también se valora mucho tener maña en las cosas prácticas, tener destreza para hacer las cosas. (Cristian, Auxiliar de Enfermería).}

En segundo lugar, la experiencia en la FP provoca una transformación no sólo en las formas de vinculación del alumnado con los aprendizajes sino también en su forma de 'estar' en el aula. En este sentido, el estigma del 'liante' se suaviza. Si bien un número importante de los entrevistados describe el entorno educativo de la FP como 'movido' o 'ruidoso' por las características mayoritarias del alumnado que escolariza, no es menos cierto que también consideran que se trabaja bien y en buen ambiente. Su vinculación con la escuela adopta una forma no convencional, es decir, que no necesariamente encaja con una imagen de la 'normalidad escolar' caracterizada por el orden, el silencio o la quietud. A pesar de sus actitudes desafiantes y hasta disruptivas, estos alumnos sienten pertenencia al centro, están comprometidos con los estudios y dotan de sentido a su presencia en el aula. Es especialmente interesante la forma como las entrevistas vinculan la conducta en el aula al interés que despiertan los aprendizajes y a la idea de 'saber qué se quiere'. Lo vemos en la siguiente cita:

\section{Algunos tienen pasado liante, pero aquí estamos los que queremos estudiar; todos estamos aqui por algo, muchos vienen de lejos y se tienen que sacrificar para estar aqui. (Edgar, Instalaciones Eléctricas y Automáticas).}

Las palabras de Edgar remiten a un elemento recurrente en las entrevistas y que tiene que ver con las relaciones que el alumnado establece con sus pares. En este sentido, son frecuentes las menciones a la heterogeneidad de las edades del alumnado de Grado Medio de FP. No obstante, estas diferencias no parecen dificultar un sentimiento de pertenencia al grupo pues este se basa, precisamente, en el 
interés común que tienen los alumnos por terminar con éxito su formación. Así, los elementos por los que se vinculan a sus pares no tienen tanto que ver con lo que les hace iguales o diferentes en términos de edad, trayectoria, género u origen, sino con lo que tienen en común respecto a sus intereses y aspiraciones. Estos intereses, por su parte, se definen en oposición a una 'cultura general' que atribuyen a la vía académica. De hecho, esta atribución pone de manifiesto el rol subsidiario que juega el conocimiento práctico en el ideario de los jóvenes -y en la estructura de la ESO-, y que lo sitúa por fuera, y no como parte de, la cultura general. Además, se considera que estos intereses denotan una idea clara de lo que quieren hacer, son específicos y están ligados al mundo real.

\section{Yo creo que tiene más valor la gente que se ha puesto a hacer FP porque es como que ya tiene claro lo que quiere hacer. (...) En cambio, con el Bachillerato estás metido en un libro y no sabes nada del mundo que hay a fuera. (Damián, Atención a Personas en Situación de Dependencia).}

La cita de Damián introduce un tercer elemento del proceso de modificación de la propia imagen de aprendiz que es, precisamente, la conexión entre la especificidad de los estudios y lo que les motiva o quieren estudiar, normalmente como vía de acceso al mercado de trabajo. En este sentido, el valor instrumental que atribuyen a la FP es mucho mayor del que asignan al Bachillerato, pues les permite acceder al mundo laboral en menos tiempo y con mayor cualificación relativa de la que tendrían si accedieran al acabar el itinerario académico:

\section{En si el Bachillerato no te abre tantas puertas, te pondrás a trabajar verdaderamente de lo que te gusta a los veinte y pico. (...) En los Ciclos, haciendo un año o dos, antes de los 18 puedes estar haciendo las prácticas y a los 18 trabajar de lo que te gusta. (Ángela, Auxiliar de Enfermería).}

Como hemos visto, el modelo de enseñanza-aprendizaje de la FP pone en el centro - con mayor o menor éxito- la vinculación con el mundo del trabajo. A pesar del valor instrumental que el alumnado atribuye a la FP, la conexión entre gustos, estudios y mundo laboral no está exenta de tensiones. Es decir, aunque la entrada a la FP puede haber mejorado los elementos positivos a través de los cuales el alumnado construye su identidad de aprendiz, sus discursos sobre el contenido explícito de la formación y sus expectativas de entrada al mercado de trabajo son a menudo críticos. La siguiente cita nos ayuda a entrever algunas de las tensiones que emergen, por un lado, de una visión dicotómica del conocimiento teórico y práctico, y, por el otro, de la conexión entre la propia formación y el tipo de acceso al mercado de trabajo que se prevé:

Me gusta cuando hacemos prácticas porque me gusta tratar con la gente, pero el estudio me da mucho palo, que si las ulceras, que si no sé qué, cosas que no vamos a hacer, porque con perdón de la palabra, vamos a acabar limpiando culos y siendo las limpias de los médicos. $Y$ aqui nos enseñan como si fueras a ser igual que las enfermeras y no, entonces a mi no me gusta sentirme que me engañen. Porque me están engañando. (Lorena, Auxiliar de Enfermería). 
Las tensiones reflejadas en las palabras de Lorena apuntan a dos elementos críticos en relación con el -potencial-impacto de la resignificación de la identidad del aprendiz sobre la trayectoria educativa del alumnado de FP y que concuerdan con los resultados de investigaciones previas como las de Colley et al. (2003) o Brockmann (2010). El primer elemento tiene que ver con la herencia de la dicotomía entre teoría y práctica que la FP recibe de las etapas previas, y especialmente de la ESO y de la forma como se acompañan las transiciones a la postobligatoria. El rechazo casi normativo a la teoría (Brockmann, 2010) en la nueva identidad de aprendices de los estudiantes de FP es sin duda un elemento que limita sus oportunidades y trayectorias educativas futuras. Y el segundo remite a las precarias condiciones en que los titulados de Grado Medio se insertan habitualmente en un mercado de trabajo cada vez más segmentado, incierto y precarizado. Parecería, pues, que la FP se encarga de educar al cada vez mayor número de 'trabajadores desanimados' que hace años que autores como Raffe (1992) o Biggart y Furlong (1996), entre otros, definen en sus investigaciones.

En este sentido, el habitus profesional que se construye en esta etapa, y que sirve de vínculo entre las experiencias formativas pasadas y presentes y las proyecciones de futuro, juega un rol ambivalente respecto a las posibilidades de emancipación o ruptura. Por un lado, la identificación con una cultura profesional posibilita la vinculación educativa a un tipo de alumnado que tradicionalmente ha estado excluido en la institución escolar, en parte por su lejanía respecto al conocimiento abstracto. Sin embargo, y precisamente por ello, refuerza una visión dicotómica y jerárquica entre ambos tipos de conocimiento, y una identificación del alumnado de FP con uno de ellos -el práctico- más que con el otro. Si bien el alumnado de FP reconstruye su identidad en esta etapa de una forma más positiva, el reconocimiento de la situación en que va a incorporarse al mercado de trabajo y, en cierta medida, su resignación, sugieren que el habitus profesional puede jugar un papel más reproductor que emancipador.

\section{Conclusiones}

El objetivo del artículo ha sido analizar cómo construyen su identidad de aprendices los alumnos de FP en base a sus experiencias educativas, pasadas y presentes, y a sus proyecciones de futuro. Siguiendo las aportaciones de autores como Brockmann (2010), Colley et al. (2003) o Ecclestone (2007a), hemos partido de la hipótesis de que esta identidad se construye en relación con experiencias educativas concretas que se generan en contextos institucionales específicos. También hemos afirmado que es fundamental analizar las identidades de los jóvenes para entender cómo se desarrollan sus trayectorias educativas. Como afirma Ecclestone (2007b) el concepto de identidad, entendido como sentido de uno mismo en relación con el mundo y con los otros, permite analizar los itinerarios y transiciones educativas más allá de miradas excesivamente dicotómicas sobre la agencia (en tanto que capacidad para la acción autónoma individual) y la estructura (en tanto que efectos de factores que constriñen o posibilitan).

A pesar de ello, hemos argumentado que todavía es escasa la investigación que analiza la identidad de los estudiantes, y en particular su identidad de aprendices, como parte de un proceso de devenir (Colley, et al., 2003), incorporando el tiempo y el cambio en su análisis. Es más, hemos afirmado que, si bien numerosas investigaciones exploran el efecto de los elementos estructurales sobre la construcción de identidades (clase, género y etnicidad, fundamentalmente), muchas menos se centran en el impacto de los elementos propiamente escolares en la construcción de las mismas (Coll y Falsafi, 2010). Dicho de otro modo, numerosos estudios demuestran cómo los ejes de desigualdad social contribuyen a construir una 
noción de uno mismo que es reconocida y/o potenciada por el contexto escolar (Archer, y Yamashita, 2003; Ingram, 2018; Reay et al., 2001). Sin embargo, son más infrecuentes las investigaciones que dan vuelta a la ecuación. Es decir, que analizan cómo los contextos escolares contribuyen a crear imágenes de uno mismo a partir de actividades y relaciones de aprendizaje específicas que, inevitablemente, están mediadas por los ejes de desigualdad.

Las principales conclusiones de nuestro análisis son las siguientes:

En primer lugar, las identidades de los jóvenes como aprendices, como hemos dicho, emergen en contextos institucionales específicos que están marcados por relaciones de poder y desigualdad. Hemos visto que la mayoría de estudiantes entrevistados parten de una experiencia negativa de la ESO, marcada por una trayectoria de notas bajas, suspensos, repeticiones y grupos adaptados, y que les dificulta construir una imagen de sí mismos como 'buenos estudiantes'. Esta experiencia, además, se nutre en el presente de la participación en un itinerario formativo, la FP, que sigue estando fuertemente desprestigiado. Si eligieron FP es porque no eran suficientemente ‘buenos' o ‘disciplinados' para estudiar Bachillerato. Esta imagen de sí mismos se ha ido gestando progresivamente a lo largo de toda su trayectoria escolar: Grupos de nivel durante la ESO con diferentes consecuencias para el acceso a la educación postobligatoria; prácticas de orientación que sitúan a la FP como un espacio para los 'no válidos'; o discursos docentes que les hacen saber que ellos 'no son buenos para estudiar'; estos elementos son algunos de los que estructuran su experiencia durante la ESO y que articulan una identidad de aprendices que, como hemos afirmado, está fuertemente deteriorada (Hollingworth y Archer, 2010).

En segundo lugar, los resultados de nuestro análisis permiten afirmar que la FP es un contexto educativo fundamental para que los jóvenes reconstruyan su propia identidad de aprendices y sus relaciones con la institución educativa. Utilizando la expresión de Ecclestone (2007a) hemos definido este espacio formativo como una 'zona de confort' para los jóvenes 'expulsados' de la ESO. Hemos argumentado que un elemento central para este proceso de reconversión es un modelo de enseñanza y aprendizaje donde el conocimiento práctico o aplicado tiene un lugar central en las prácticas pedagógicas y curriculares. De hecho, dar valor a lo práctico se convierte para los jóvenes en un 'acto de reconocimiento' (Coll y Falsafi, 2010) de lo que entienden como elemento constitutivo de su forma de ser y de aprender, y que hasta ahora había sido ninguneado. $\mathrm{Y}$ es desde aquí desde dónde pueden reconstruirse como aprendices y desarrollar lo que Colley et al. (2003) definen como un habitus profesional.

En tercer lugar, nuestro análisis muestra que este proceso de reconstrucción de la identidad de aprendiz no está exento de contradicciones y que, aunque con intención de cambio o transformación (utilizando la expresión clásica de Willis, 1977), acaba teniendo un efecto de reproducción. Ciertamente, reclamar el valor de lo práctico como forma de conocimiento puede interpretarse como un acto de resistencia. No son ellos los que no son 'buenos estudiantes', es el sistema educativo que no reconoce sus habilidades. El mismo hecho de nombrar al conocimiento práctico como conocimiento ya es un acto de poder que les permite significarse a sí mismos como aprendices. Sin embargo, las dicotomías entre teoría y práctica que atraviesan el sistema educativo obligatorio y postobligatorio en nuestro país son un reflejo de la estructura de desigualdad social y, en particular, de la división social del trabajo Tarabini y Jacovkis, 2020). El rechazo de la teoría como elemento constitutivo de sus nuevas identidades de aprendices permite a los estudiantes reconciliarse consigo mismos y con la escuela en el presente, pero les cierra puertas en el futuro. Más aún, teniendo en cuenta la enorme segmentación y precariedad que atraviesa el mercado laboral. 
Así pues, es fundamental que la escuela y sus profesionales reconozcan su papel crucial en la construcción de las identidades de aprendices de los jóvenes (Coll y Falsafi, 2010; Reay, 2010). Éstos tienen derecho a acceder y experimentar diferentes tipos de conocimiento; tienen derecho a sentirse reconocidos, como personas y como aprendices; tienen derecho a ser escuchados; y tienen derecho también a ser queridos y cuidados en el marco de la escuela. Porque, como hemos argumentado, la experiencia del fracaso escolar es profundamente emocional e impacta en el presente y en el futuro de los jóvenes.

\section{Referencias bibliográficas}

Archer, Louise y Francis, Becky (2007). Understanding Minority Ethnic Achievement: Race, Gender, Class and 'Success'. London: Routledge

Archer, Louise y Yamashita, Hiromi (2003): “Knowing their limits? Identities, inequalities and inner city school leavers' post-16 aspirations”. Journal of Education Policy, 18 (1): 53-69. DOI: https://doi.org/1 $0.1080 / 0268093032000042209$.

Ball, Stephen (1993): "What is policy? Texts, trajectories and toolboxes". Discourse, 13 (2),10-17.

Ball, Stephen; Maguire, Meg y Macrae, Sheila (2000). Choice, Pathways and Transitions post-16: New youth, New Economies in the Global City. London: Routledge.

Biggart, Andy y Furlong, Andy (1996): “Educating 'Discouraged Workers': Cultural Diversity in the Upper Secondary School”. British Journal of Sociology of Education, 17 (3), 253-26.

Coll, César y Falsafi, Leili (2010): "Learner Identity. An Educational and Analytical tool”. Revista de Educación, 353, 211-233.

Brockmann, Michaela (2010): "Identity and Apprenticeship: The Case of English Motor Vehicle Maintenance Apprentices". Journal of Vocational Education and Training, 62(1), 63-73. DOI: https://doi. org/10.1080/13636820903461354.

Colley, Hellen; James, David; Diment, Kim y Tedder, Michael (2003): “Learning as Becoming in Vocational Education and Training: Class, Gender and the Role of Vocational Habitus". Journal of Vocational Education and Training, 55 (4): 471-97. DOI: https://doi.org/10.1080/13636820300200240.

Ecclestone, Kathryn (2007a): "Commitment, Compliance and Comfort Zones: The Effects of Formative Assessment on Vocational Education Students' Learning Careers". Assessment in Education, 14 (3), 315-33. DOI: https://doi.org/10.1080/09695940701591925.

Ecclestone, Kathryn (2007b): Lost and Found in Transition: the Implications of 'Identity', 'Agency' and 'Structure' for Educational Goals and Practices. Keynote presentation to Researching Transitions in Lifelong Learning Conference, Univerisity of Stirling, 22-24 June 2007

Ingram, Nicola (2018). Working-Class Boys and Educational Success Teenage Identities, Masculinities and Urban Schooling. Palgrave: London.

Harris, Roger y Rainey, Linda (2012): "Learning Pathways Between and Within Vocational and Higher Education: Towards a Typology?”. The Australian Association for Research in Education, 39, 107-123. DOI: https://doi.org/10.1007/s13384-012-0052-1 
Furlong, John Cardiff (1991): "Disafected Pupils: Reconstructing the Sociological Perspective". British Journal of Sociology of Education, 12 (3), 293-307.

García Jiménez, Enrique y Lorente García, Rocío (2015). "Recorrido por la imagen social de la formación profesional: un camino hacia su revalorización”. Revista Española de Educación Comparada, 26, 119-134. DOI: https://doi.org/10.5944/reec.26.2015.14270.

Grant, Barbara (2006). "Disciplining Students: the Construction of Student Subjectivites". British Journal of Sociology of Education, 18 (1), 101-114. DOI: https://doi.org/10.1080/0142569970180106.

Hodkinson, Phil; Biesta, Gert y James, David (2007) "Understanding Learning Cultures". Educational Review, 59 (4), 415-427. DOI: https://doi.org/10.1080/00131910701619316.

Hollingworth, Sumi y Archer, Louise (2010): “Urban Schools as Urban Places: School Reputation, Children's Identities and Engagement with Education in London". Urban Studies, 47 (3), 584-603. DOI: https://doi.org/10.1177/0042098009349774.

Jenkins, Ricard (1996). Social Identity. London: Routledge.

Nylud, Mattias y Rosvall, Per-Åke (2016): “A Curriculum Tailored for Workers? Knowledge Organization and Possible Transitions in Swedish VET”'. Journal of Curriculum Studies, 48 (5): 692-710. DOI: https:/ / doi.org/10.1080/00220272.2016.1138325.

Raffe, David (1992): "Participation of 16-18 Year Olds in Education and Training". National Commission on Education, Briefing Paper, 3. London, National Commission on Education.

Reay, Diane (2010): “Identity Making in Schools and Classrooms” en Margaret Wetherell y Chandra Mohanty (eds.): The SAGE Handbook of Identities. London: Sage publications.

Reay, Diane; Davies, Jaqueline; David, Miriam; Ball, Stephen (2001): “Choices of Degree or Degrees of Choice? Class, Race and the Higher Education Choice Process”. Sociology, 35 (4): 855-874. DOI: https://doi.org/10.1177/0038038501035004004.

Rees, Gareth; Fevre, Ralph; Furlong, John Cardiff y Gorard, Stephen (1997). "History, Place and the Learning Society: Towards a Sociology of Lifetime Learning”. Journal of Education Policy, 12 (6): 485497, DOI: https://doi.org/10.1080/0268093970120604.

Romito, Marco (2017): “Governing Through Guidance: An Analysis of Educational Guidance Practices in an Italian Lower Secondary School". Discourse: Studies in the Cultural Politics of Education, 40 (6): 773 788. DOI: https://doi.org/10.1080/01596306.2017.1314251.

Tarabini, Aina y Jacovkis, Judith (2019): “QQué conocimiento para quién? Itinerarios escolares, distribución del conocimiento y justicia escolar”. e-Currículum, 17 (3): 880-908. DOI: https://doi.org/10.2392 5/1809-3876.2019v17i3p880-908.

Tarabini, Aina y Jacovkis, Judith (2020). "Las transiciones a la educación secundaria post obligatoria en Cataluña: un análisis desde la sociología de la Política Educativa". Archivos Analíticos de Políticas Educativas, 28 (102), 1-25. . 
Valenzuela, Angela (1999). Subtractive Schooling: US-Mexican Youth and the Politics of Schooling. Albany: State University of New York Press.

Willis, Paul (1977). Learning to Labour: How Working Class Kids Get Working Class Jobs. London: Saxon House.

Youdell, Deborah (2003): "Identity Traps or How Black Students Fail”. British Journal of Sociology of Education, 24 (1): 3-20. DOI: https://doi.org/10.1080/01425690301912.

Wheelahan, Leesa (2007). "How Competency-Based Training Locks the Working Class out of Powerful Knowledge: A Modified Bernsteinian Analysis". British Journal of Sociology of Education, 28 (5), 637-651. DOI: https://doi.org/10.1080/01425690701505540.

\section{Notas biográficas}

Aina Tarabini es Doctora en Sociología por la Universidad Autónoma de Barcelona, profesora de sociología en la misma universidad e investigadora de los grupos de investigación GEPS (Globalización, Educación y Política Social) y GIPE (Grupo Interdisciplinar de Políticas Educativas). Es especialista en sociología de la educación y en análisis de desigualdades y políticas educativas. Investiga sobre fracaso y abandono escolar, transiciones educativas y prácticas pedagógicas desde la perspectiva de la justicia social. Actualmente es la investigadora principal del proyecto I+D+I 'Edupost16. La construcción de las oportunidades educativas post-16. Un análisis de las transiciones a la educación secundaria post-obligatoria en contextos urbanos'.

Orcid: https://orcid.org/0000-0002-6096-2450.

Judith Jacovkis es Doctora en Sociología por la Universidad Autónoma de Barcelona. Ha participado en distintas investigaciones sobre políticas sociales de transferencia de renta, desigualdades y políticas educativas especialmente orientadas a población en situación de vulnerabilidad. Actualmente está centrada en el estudio de las políticas, la gobernanza y la experiencia de las trayectorias y transiciones educativas a través de los proyectos «Can dual apprenticeships create better and more equitable social and economic outcomes for young people? A comparative study of India and Mexico» y «EDUPOST16 La construcción de las oportunidades educativas post-16. Un análisis de las transiciones a la educación secundaria post-obligatoria en contextos urbanos». Además, es investigadora del proyecto «ECASS. European Cities Against School Segregation».

Orcid: https://orcid.org/0000-0003-2862-8636.

Marta Curran es Doctora en Sociología por la Universidad Autónoma de Barcelona y actualmente profesora e investigadora de Sociología de la Educación en la Universidad Complutense de Madrid. Miembro del grupo de investigación Globalización, Educación y Políticas Sociales de la UAB. Su investigación se centra en el análisis de las desigualdades en educación, el análisis de políticas y las transiciones educativas. . Orcid: https://orcid.org/0000-0003-1956-7616. 